\title{
The Importance of Polyphenols as Functional Food in Health
}

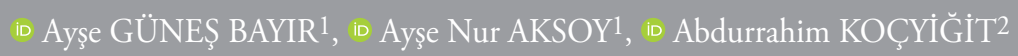

1Bezmialem Vakıf University Faculty of Health Science, Department of Nutrition and Dietetics, İstanbul, Turkey

${ }^{2}$ Bezmialem Vakıf University Faculty of Sciences, Department of Medical Biochemistry, İstanbul, Turkey

\begin{abstract}
Polyphenols which are produced by plants are very important functional foods in our nutrition. Because of their diverse chemical structures, they are subject to main classification among them. The main groups of polyphenols are; flavanoids, lignans, stilbenes and phenolic acids. At the present time, the health effects of them have been investigated in several in vivo and in vitro studies. In addition to their antioxidant effects, they are known to have a pro-oxidant character. Polyphenols have important effects in protecting the body against external factors and the cleansing of reactive oxygen species which occur as a consequence of some diseases. Furthermore, polyphenols have effects on protection from specific diseases and stop their progression with certain mechanisms. In this review, various effects of polyphenols including antioxidant, pro-oxidant, cytotoxic, anti-inflammatory, antihypertensive, anti-diabetic are discussed which obtained from in vitro, in vivo experimental animal and clinical investigations.
\end{abstract}

Keywords: Polyphenols, functional food, antioxidant, pro-oxidant, biological activities

\section{Introduction}

Plant-based foods in our diet consist of primary and secondary ingredients produced by plants. Plants synthesize secondary metabolites such as terpenes, saponins, glycosides and polyphenolic compounds that are not present in all living things as well as primary metabolites such as carbohydrates, proteins and fats in order to maintain their growth and liveliness (1). Most of the secondary metabolites are consisted of polyphenols. Polyphenols make up most of the components found in all vegetative organs, fruits and flowers. Plants produce polyphenols as secondary metabolites to protect themselves and interact with other plants. Also, polyphenols have an effect on the formation of bitter taste in plants.

\section{Chemical Structure And Classification of Polyphenols}

Polyphenols contain at least one aromatic ring and one or more hydroxyl groups, in addition to other components of them
(2). According to their chemical structure, they are classified as follows: Flavanoid, Lignan, Stilben and phenolic acids. The main classification of polyphenols is shown in Figure 1.

\section{Flavonoids}

All flavonoid species are formed as a result of the biosynthesis of shikimic acid and acetate-malonate pathways together (3). Flavonoid subspecies: a) Flavonol, b) Flavanol, c) Isoflavon, d) Flavon, e) Flavanon, f) Anthocyanin (4).

a) The richest sources of flavonols are onion $(1.2 \mathrm{~g} / \mathrm{kg}$ of wet weight), broccoli, leeks and blueberries. Tea and red wine contain about $45 \mathrm{mg} / \mathrm{L}$ flavonols. In general, these components are glycosylated with glucose and ramnose. Five to ten kinds of flavonol glycosides are in the content of fruits. For biosynthesis of flavonols in plants, exposure to sunlight is required. Greenish leaves of leafy vegetables such as lettuce and cabbage have 10 times more flavonol glycosides than the light-colored leaves inside (4).

Address for Correspondence: Ayşe GÜNEŞ BAYIR, Bezmialem Vakıf University Faculty of Sciences, Department of Nutrition and Dietetics, İstanbul, Turkey 


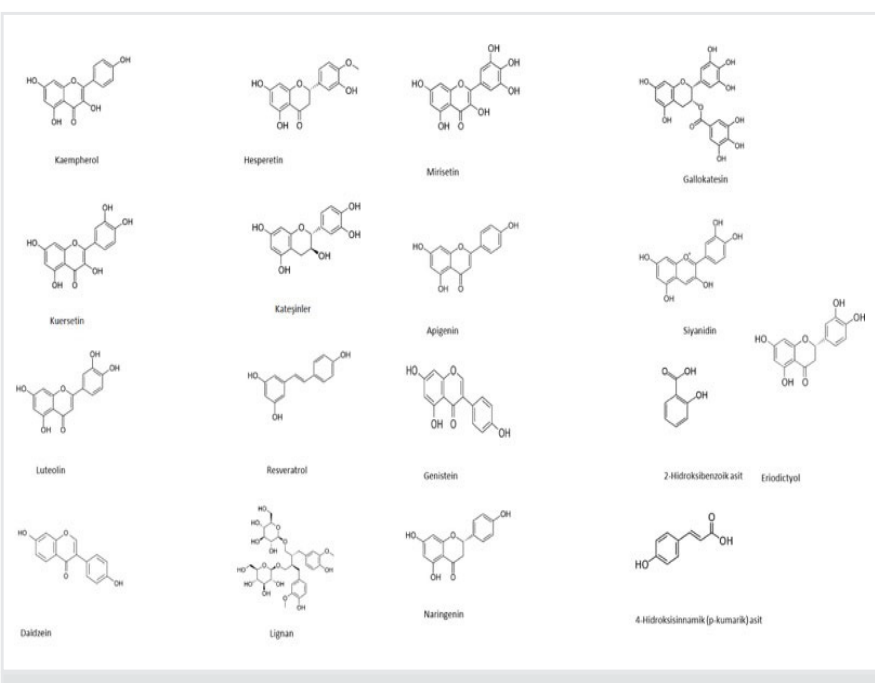

Figure 1. General classification of polyphenols

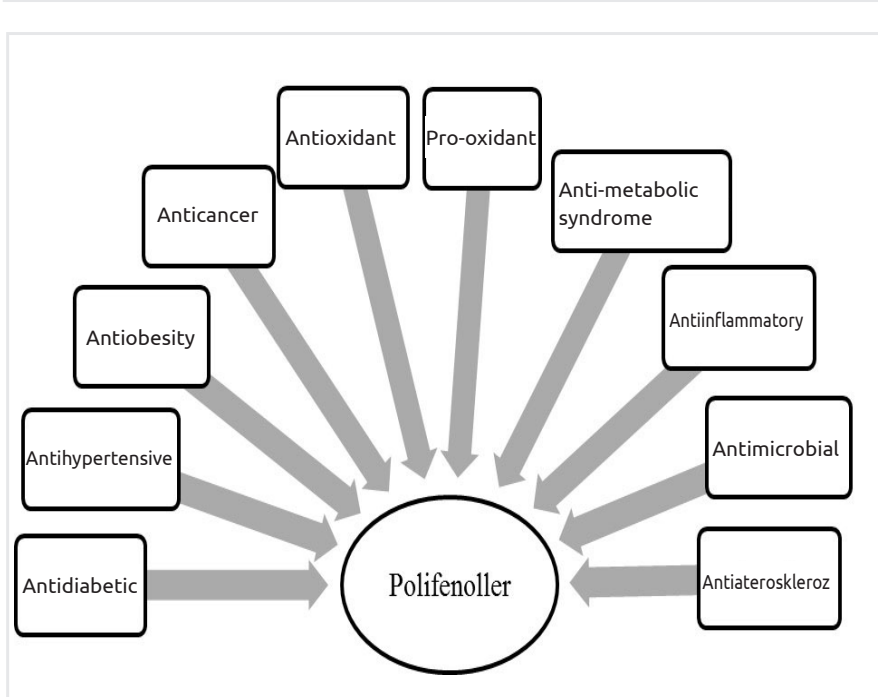

Figure 2. Biological activity of polyphenols

b) Flavanols have two forms: Catechin in monomer structure and proanthocyanidin in polymer structure. Most catechins are found in fruit species. The richest sources are green tea, cocoa and red wine. However, black tea contains less flavanol in monomer form because green tea is oxidized during fermentation but is rich in theaflavin and thearubigine which are polyphenols in polymer form (4).

c) Isoflavones are similar to estrogen as chemical structure and are most commonly found in soybean products. Because it binds to estrogen receptors in the body, it is classified as phytoestrogen. It usually contains three main molecules: Genistein, daidzein and glisitein (4).

d) Flavones are most commonly found in dry tea leaves (especially chamomile), parsley, celery, chicory (5).

e) Flavanones are found in citrus fruits with the highest concentrations. The most important flavanones are hesperidin, naringen and eriodictyol. The major flavanone glycosides in orange (Citrus sinensis) are narirutin, a derivative of hesperidin and naringenin (6).

f) Anthocyanins are found in eggplant, red fruits, cherry, strawberry and black grape (4). Anthocyanins are dominant pigments that give plants the color of red, purple and purple pink. The pigmentation of flowers is determined by the type and amount of anthocyanin in the contents of the flower (7). When lingonberry (Vaccinium vitis-idaea) which is grown in Poland and blueberries (Vaccinium myrtillus L.) are compared in terms of phenolic ingredients they contain, blueberries have shown more antioxidant activity. It was found that increased anthocyanin content in blueberry increased the effect of radical cleansing and antioxidant activity (8).

\section{Lignans}

Flax seed is the richest source of lignan called secoisolariciresinol diglycoside (SDG). The lignan complex isolated from flax seed contains 34-38\% SDG, $15-21 \%$ sinnamic acid glucoside and 9.6-11\% hydroxymethyl glutaric acid. It is metabolized by anaerobic intestinal microorganisms and converted to lignans called enterolactone and enterodiol (9).

\section{Stilbens}

The most common of stilben subtypes is resveratrol. It is found in grapes, red wine and some forest fruits. It is very limited to use in the body because it is taken in a very little amount in daily diet. However, recent studies were carried out to increase bioavailability of resveratrol in the body and to understand the various functions of resveratrol. It is known that it has anticancer effect and has remarkable properties in the treatment of neurodegenerative diseases (10).

\section{Phenolic Acids}

Phenolic acids are divided into 2 types: 1-) Benzoic acid and derivates, 2-) Cinnamic acid and derivates

a) Benzoic acid and derivates: The concentration of hydroxybenzoic acid in plants is usually very small, a certain amount of it is found in red fruits, onion and black radish (4).

b)Cinnamic acid and analogues: They are caffeic acid, ferulic acid and p-coumaric acid. Hydroxycinnamat, the major phenolic substance in the cherry, is found at highest concentration in the outer membrane of the cherry (11).

\section{Bioactivities of Polyphenols}

Polyphenolic compounds are known to have many biological activities. The most common features of polyphenols with a wide range of biological activities are their antioxidant and prooxidant characters (12). Figure 2 shows the biological activities of polyphenols. The potential role of polyphenols in preventing and/or treating chronic diseases such as cardiovascular and neurodegenerative diseases and cancer is extremely important which (13). 


\section{Anti-oxidant Activity}

Reactive oxygen species (ROS) are characterized by a nonstable high-energy oxygen atom containing a double-formed electron in the outer orbitals (14). ROS are mainly composed of superoxide radicals $\left(\mathrm{O}_{2}^{-}\right)$, hydrogen peroxide $\left(\mathrm{H}_{2} \mathrm{O}_{2}\right)$ and hydroxyl radicals $\left(\mathrm{OH}^{-}\right)$. ROS production occurs in most aerobic organisms. Ultraviolet rays around us, radiation, cigarette smoke, aging, pathogenic invasion are factors that increase the formation of ROS $(15,16)$. Low doses of ROS in the body play a role in cell signal transmission. However, excessive ROS cause some harmful effects. For example, oxidative stress occurs as a result of the imbalance between ROS production and metabolism. However, the development of oxidative stress and its effect on the cell depends mainly on the ability of the organism to maintain its dynamic balance of redox state (16). As ROS accumulate, the function of cells and tissues deteriorates and this accumulation causes various conditions such as cancer, atherosclerosis, and neurodegenerative diseases $(17,18)$. ROS production occurs in plants as well as animals. Plants produce ROS to protect themselves against pathogens and external stress. Plants and animals use similar systems to respond to ROS (19). The human body needs endogenous antioxidants as well as exogenous antioxidants to minimize effects of ROS. In this respect, polyphenols that show antioxidant activity are extremely important in terms of human health (15). Polyphenols protect cells and tissues against oxidative damage by acting as antioxidants. They do this by cleaning radicals and by protecting DNA from oxidative damage and LDL from peroxidation (12).

Low doses of phenolic terpens called carvacrol in essential oils of plants from the Lamiaceae family were shown to play an important role in the prevention of diseases such as cancer due to their antioxidant activity (20).

The drug, cisplatin (CP), used in the treatment of cancer, has toxic effects on liver, kidney and genes. In a study conducted on rats, it was shown that when rats were first treated with naringenin-oxime (NOX) which is produced from naringenin and then $\mathrm{CP}$ was injected to rats, NOX showed an antioxidant effect and reduced the oxidative stress and DNA damage (21).

\section{Pro-oxidant Activity}

Polyphenols are often known for their antioxidant structure. However, high doses of some polyphenolic compounds were shown to cause DNA damage, apoptosis, and cell death. $(12,20)$. Free radicals damage DNA, lipids and proteins in the cell. Phenoxyl radicals, which are produced during cleaning of free radicals by some plant polyphenols, oxidize proteins and lipids and thus show pro-oxidant activity. Flavonoids and tannic acid, for example, cause DNA degradation due to the formation of hydroxyl radicals as a result of Fenton Reaction in the presence of copper (II) $(12,22)$. In addition, some flavonoids may show prooxidant activity in the presence of a free radical, $\mathrm{NO}$ (nitric oxide), and cause DNA damage $(12,23)$. Although tea polyphenols are known as antioxidants, there is evidence that these components have pro-oxidant activity. High dose tea polyphenols show prooxidant activity in hepatocytes and cause toxicity (24). High doses of polyphenols may inhibit carcinogenesis as a result of their effects that initiate apoptosis. Carvacrol and tea polyphenols increase ROS in cancer cells with a dose-dependent manner which is a sign of their pro-oxidant activity $(16,20)$. Therefore, the dose of antioxidants in the treatment is extremely important.

\section{Anti-cancer Effect}

Since the normal level of antioxidant defense system in the cell is often insufficient to neutralize free radicals of endogenous or exogenous origin, there is a need for antioxidants of exogenous origin (25).

Polyphenols have a protective effect against certain types of cancer. They inhibit the growth of tumors, and on the other hand they have cytotoxic effects on cancer cells, induce apoptosis in cancer cells and stimulate the activity of various enzymes (25). Cytotoxic effects of polyphenolic compounds on cancer cells were found to be associated with their pro-oxidant activities. Prooxidant activity is related to the dose and molecular structure of polyphenolic compounds, oxidative state of the environment and the presence of free iron and copper.

An in vitro study on human breast cancer cells revealed that, between 5 flavonoids, myricetin showed the highest cytotoxic effect in the presence of copper ions. This suggests that the chemical structure of the polyphenols is different and that their cytotoxic effects on cancer cells are different (22).

In an in vitro study, the effect of polyphenols which are abundant in grape seed and shell contents on human epidermoid carcinoma cells was investigated. According to the results, grape seed and shell stimulated apoptosis in skin cancer cells and caused various morphological changes. Therefore, alkaloids, flavonoids, saponins and tannins in grape content are of therapeutic importance (25). Curcumin, the main active ingredient in turmeric, which has been used as a spice for many years, is a pigment and phenol that stands out with its anti-cancer and anti-inflammatory properties. Curcumin inhibits the division of tumor cells and increases the rate of death of cancer cells in some types of cancer. For example, in clinical trials performed on curcumin, it was determined that it was effective on breast, pancreas, and stomach cancers (26). Tea and tea polyphenols have a protective and therapeutic effect on many organs. The potential antioxidant effect of tea polyphenols was observed in different animal carcinogenesis models. In these models polyphenols inhibit carcinogenic activity by activating various enzymes such as detoxifying glutathione $S$-transferase and superoxide dismutase, and they prevent the onset of cancer. According to results obtained from in vivo experiments, tea and tea polyphenols are antioxidants, as well as they inhibit colonization of tumor-initiating cells. Thus, it restricts the progression of carcinogenesis to early dysplastic stage in the treatment process (27).

\section{Anti-atherosclerotic Effect}

Polyphenols from various plant foods have a protective effect on cardiovascular system by various mechanisms (preventing oxidation of LDL, increasing HDL, reducing LDL and triglycerides). The most studied herbal foods with anti- 
atherosclerotic proporties are; extra virgin olive oil, bitter chocolate, black and green tea, forest fruits, citrus fruits and grapes (28). LDL, which is made up of lipophilic structures, is one of the causes of atherosclerosis when oxidized. Several studies have shown that some polyphenols protect LDL from oxidation (4). Olive oil polyphenols protect LDL from oxidation and also contribute to down regulation of gene expression that causes it (29). It is reported that resveratrol (30) and tannic acid (31) have anti-atherosclerotic effects by improving endothelial dysfunction in cell culture studies and animal and human experiments.

\section{Anti-metabolic Syndrome}

Metabolic syndrome is a condition caused by the clustering of cardiometabolic factors such as dyslipidemia, high blood pressure, blood sugar regulation disorder, abdominal obesity (32). The possible mechanism of influence of polyphenols on metabolic syndrome is to improve these factors.

In a study of Polish adults, the association between daily polyphenol intake and prevalence of metabolic syndrome was examined. When daily polyphenol intake was grouped according to age, taken total energy and gender, significant results were achieved (33). The body mass index (BMI), waist circumference (WC), blood pressure and triglyceride levels were significantly lower in individuals taking more polyphenols, while a linear correlation was found only with WC and BMI. High polyphenol intake in women was negatively correlated with blood pressure, high LDL cholesterol and triglyceride levels, whereas high polyphenol intake in both genders was negatively correlated with fasting plasma glucose. Polyphenols including phenolic acid and stilben are significantly associated with metabolic syndrome. It was found that lignans and stilbens were associated with WC, phenolic acids with blood pressure and triglycerides and flavonoids with fasting plasma glucose (33).

Basu et al. randomized 35 individuals with obesity and metabolic syndrome into groups consisted of 3 individuals based on age and gender in a randomized controlled clinical trial. Water (4 cups/ day), green tea ( 4 cups/day) and green tea extract ( 2 capsules/ day) were randomly given to individuals in the groups for 8 weeks. In binary comparisons, weight loss and reduce in body fat mass in individuals who consumed green tea or green tea extract compared to control group (34).

Curcumin has a significant protective effect on fatty liver disease, which can develop due to the fat dysfunction seen in the metabolic syndrome. In a prospective cohort study of 100 patients with metabolic syndrome, patients were given 400 mg of curcumin per day for one year. At the end of the study, morphological changes in liver were visualized by ultrasound and improvement was observed (35).

\section{Anti-obesity Effect}

Polyphenols affect obesity by inhibiting pre-adipocyte differentiation, reducing adipocyte proliferation, stimulating adipocyte apoptosis, suppressing lipogenesis and contributing to lipolysis and beta oxidation of fatty acids in in vitro conditions.
The anti-obesity effect of epigallocatechin gallate (EGCG) and green tea extract has been shown in cell culture, animal and human models $(34,36)$. On the other hand, EGCG reduces the release of resistin, an inflammatory adipokine, which is a derivative of adipocyte. Resistin is also associated with insulin resistance and cardiovascular disease risk. Because visceral fat stores contribute to chronic inflammation in obesity, it is important to reduce oxidative stress and ROS molecules (37). In this regard, since green tea acts as an antioxidant, it can be effective in reducing the destructive effect of ROS molecules and in suppressing the inflammation in the development of obesity (10).

\section{Anti-hypertensive Effect}

Cocoa is a plant that is extensively searched in scientific interventional research on human beings. Cocoa beans contain polyphenolic component up to $6-8 \%$ of their dry weights. Cocoa accommodates plenty of flavonols, especially epicatechin, catechin and procyanidin within. It is known that flavanolrich cocoa products improve endothelial function, reduce the sensitivity of LDL to oxidation, and increase endotheliumdependent vasorelaxation $(38,39)$. The effect of cocoa flavanols on endothelium-dependent vasodilation is accepted by the European Food Safety Authority ( EFSA) (38).

In a study by Grassi et al. (39), 19 hypertensive patients with glucose tolerance disorder were randomized and a group of patients were given 100 grams of bitter chocolate rich in flavanol for 15 days and the other group was given the same amount of flavanol-free white chocolate. Fifteen days later, treatments were exchanged. Insulin resistance and systolic and diastolic blood pressures decreased and insulin sensitivity increased in bitter chocolate eaters. In a meta-analysis, it was found that the quercetin was significantly effective in reducing blood pressure (40).

\section{Anti-microbial Effect}

Anti-microbial activity of polyphenols on a wide range of microorganisms is investigated. However, especially flavonoids have more anti-microbial effects than other polyphenols. In addition, most flavonoids are highly important because they suppress microbial virulence and show synergism with antibiotics (41).

In a study on thyme plant rich in polyphenols and flavonoids, the antibacterial effect of thyme extract on gram-positive bacteria (Staphylococcus and B.subtilis) and gram negative bacteria (E.coli and $P$. Aeruginosa) was investigated. According to this; thyme extract inhibited the growth of tested bacteria from medium to high level. Maximum effect was observed against Paeruginosa (42). EGCG, the tea polyphenol, has anti-microbial, antifungal and antiviral effects. It inhibits the binding of the influenza virus to the target receptor on the cell (41). In a study by Seo and Choi (43), mouse raw 264.7 macrophage cells were treated with five flavonoids including EGCG, quercetin, fisetin, daidzein and ECG. According to this, the release of antiviral agents was regulated up by all flavonoids and neuroviruses were significantly inhibited (43). Polyphenols in olive oil provide bacterial 
inhibition. Various extra virgin olive oils obtained from different geographical regions of Turkey have provided microbial inhibition of some important nutrient pathogens (E. coli O157:H7, Listeria monocytogenes and Salmonella enteritidis). However, this effect was not observed in refined olive oil, hazelnut and canola oils (44).

\section{Anti-diabetic Effect}

A diet rich in polyphenols reduces the risk of development of diabetes. Various polyphenols positively regulate insulin and (glucagon-like peptide-GLP-1) pathways. In addition, polyphenols increase insulin sensitivity in peripheral tissues (45). In a randomized and controlled study of overweight/obese adult individuals; the effect of diets rich in polyunsaturated fatty acids, in long chain omega-3 fatty acids and in both on blood glucose for 8 weeks. Foods rich in polyphenols are bitter chocolate, extra virgin olive oil, decaffeinated coffee green tea, cranberry jam and polyphenol-rich vegetables. In a study, polyphenols taken with diet reduced blood glucose levels and increased early insulin secretion in 3-hour OGTT (oral glucose challenge test) at the end of eight weeks compared with 3-hour OGTT performed before the study (46). It is known that cinnamon has many beneficial effects on hyperlipidemia and use of glucose. In a study conducted on rats, cinnamon extract reduced visceral fat mass, liver weight, serum glucose level and insulin concentration (47). Palm has gastric-protecting, anti-cancer, anti-inflammatory, and anti-hyperglycemic effects and is a very common fruit in some communities. Palm contains phenolic acids from polyphenols, lignans, isoflavones, flavonoids and tannin. In addition, it strongly inhibits the $\alpha$-glucosidase and $\alpha$-amylase enzymes and thus regulates the absorption of glucose from the intestines and contributes to the normal glucose balance. Therefore, it is a potentially effective fruit in preventing and controlling diabetes mellitus (48).

\section{Anti-inflammatory Effect}

Some plant polyphenols have effects on immune system and inflammatory cells. Some phenolic agents influence cytokine and cytokine receptors, while others affect the secretory process (12).

Chlorogenic acid is a phenolic compound formed by esterification of caffeic acid and quinic acid. This compound has been shown to have an anti inflammatory effect in various animal experiments. Ohkawara et al. (49) injected $20 \mathrm{mg} / \mathrm{kg}$ or $40 \mathrm{mg} / \mathrm{kg}$ chlorogenic acid to rats, and pancreatitis was subsequently initiated. The histological effects of pancreatitis decreased in rats given $40 \mathrm{mg} /$ $\mathrm{kg}$ chlorogenic acid. The macrophage migration inhibitory factor (MIF) level, which is a pro-inflammatory cytokine, decreased in serum and pancreas (49).

The grape peel extract, rich in Anthocyanidin, when combined with a high-fat diet on mice caused various metabolic changes (weight gain, dyslipidemia, insulin resistance, change in some hormones released from adipose tissue), but inflammatory markers such as TNF-a, IL-6 decreased significantly (50).

\section{Conclusion}

Polyphenolic compounds, which are secondary metabolites produced by plants, are a large group of molecules of different chemical structure. In addition to the effects of polyphenols on many diseases such as anti-hypertensive, anti-microbial, anti-obesity, anti-diabetic effects in humans and animals; the most remarkable and well-they antioxidant effect prevents the formation of ROS when used in low doses and prevents many diseases such as cancer. However, high doses of certain polyphenols may have a pro-oxidant effect and cytotoxic and apoptotic effects especially on cancer cells. This situation also offers the option of polyphenols for the treatment of such disease.

Peer Review: Externally peer-reviewed

\section{Authorship Contributions}

Concept: A.G.B., Design: A.G.B., Data Collection or Processing: A.G.B., A.A., Analysis or Interpretation: A.G.B., A.A., A.K., Literature Search: A.A., A.G.B., Writing: A.G.B., A.A., A.K.

Conflict of Interest: No conflict of interest was declared by the authors.

Financial Disclosure: The authors declared that this study received no financial support.

\section{References}

1. Rizvi SI, Pandey KB. Plant polyphenols as dietary antioxidants in human health and disease. Oxid Med Cell Longev 2009;2:270-8.

2. Ferrazzano GF, Amato I, Ingenito A, Zarrelli A, Pinto G, Pollio A.Plant polyphenols and their anti-cariogenic properties: A Review. Molecules 2011;16:1486-507.

3. Castellano G, Gonzalez-Santander JL, Lara A, Torrens F. Classification of flavonoid compounds by using entropy of information theory. Phytochemistry 2013;93:182-91.

4. Manach C, Scalbert A, Morand C, Remesy C, Jimenez L. Polyphenols: food sources and bioavailability. Am J Clin Nutr 2004;79:727-47.

5. Hostetler G, Ralston RA, Schwartz SJ. Flavones: Food Sources, bioavailability, metabolism, and bioactivity. Adv Nutr 2017;8:42335 .

6. Kroon PA, Brett GM, Hollands W, Needs PW, Teucher B, Dainty $\mathrm{JR}$, et al. Absorption, metabolism and excretion of flavanones from single portions of orange fruit and juice and effects of anthropometric variables and contraceptive pilluse on flavanone excretion. Br J Nutr 2009;101:664-75.

7. Du H, Lai L, Wang F, Sun W, Zhang L, Li X, et al. Characterization of flower coloration in 30 Rhododendrons pecies via anthocyanin and flavonol identification and quantitative traits. Plant Biol (Stuttg) 2018;20:121-9.

8. Dróżdż P, Šèžienè V, Pyrzynsk K. Phytochemical properties and antioxidant activities of extracts from wild blueberries and lingonberries. Plant Foods Hum Nutr 2017;72:360-4.

9. Prasad K. Flax seed and cardiovascular health. J Cardiovasc Pharmacol 2009;54:369-77. 
10. Wang S, Moustaid-Moussa N, Chen L, Mo H, Shastri A, Su R, et al. Novel insights of dietary polyphenols and obesity. J Nutr Biochem 2014;25:1-18.

11. Sotelo KAG, Hamid, N, Oey, I, Pook, C, Gutierrez-Maddox N, Ma $\mathrm{Q}$, et al. Redcherries(Prunus avium var. Stella) processed by pulsed electric field - physical, chemical and microbiological analyses, Food Chemistry 2018;240:926-34.

12. Ferguson LR. Role of plant polyphenols in genomic stability. Mutat Res 2001;475:89-111.

13. Zhou Y, Zheng J, Li Y, Xu DP, Li S, Chen YM, et al. Natural polyphenols for prevention and treatment of cancer. Nutrients 2016;8:2-35.

14. Halliwell B. Biochemistry of oxidative stress. Biochem Soc Trans 2007;35:1147-50.

15. Kocyigit A, Selek S. Eksojen Antioksidanlar iki yönü keskin kılıçlardır. Bezmialem Science 2016;2:70-5.

16. Mao X, Gu C, Chen D, Yu B, He J. Oxidative stress-induced diseases and tea polyphenols. Oncotarget 2017;8:81649-61.

17. Arouoma OI. Free radicals, oxidative stress, and antioxidants in human health and disease. J Am Oil Chem Soc 1998;75:199-212.

18. Alagawanyl M, El-Hack MEA, Farag MR, Tiwari R, Dhama K. Biological effects and modes of action of carvacrol in animal and poultry production and health - a review. Adv Anim Vet Sci 2015;3:73-84.

19. Mahalimgam R, Fedoroff N. Stress response, cell death and signalling: the many faces of reactive oxygen species. Physiol Plant 2003;119:5668.

20. Gunes-Bayir A, Kiziltan HS, Kocyigit A, Güler EM, Karatas E, Toprak A. Effects of natural phenolic compound carvacrol on the human gastric adenocarcinoma (AGS) cells in vitro. Anti-Cancer Drugs 2017;28:522-30.

21. Koyuncu I, Kocyigit A, Gonel A, Arslan E, Durgun M. The protective effect of naringenin-oxime on cisplatin-induced toxicity in rats. Biochem Res Int 2017;2017:9478958.

22. Arif H, Sohail A, Farhan M, Rehman AA, Ahmad A, Hadi SM. Flavonoids-induced redox cycling of copper ions leads to generation of reactive oxygen species: a potential role in cancer chemoprevention. Int J Biol Macromol 2018;106:569-78.

23. Ohshima H, Yoshie Y, Auriol S, Gilibert I. Antioxidant and prooxidant actions of flavonoids: effects on DNA damage induced by nitric oxide, peroxynitrite and nitroxyl anion. Free Radic Biol Med 1998;25:1057-65.

24. Galati G, Lin A, Sultan AM, O’Brien PJ. Cellular and in vivo hepatotoxicity caused by green tea phenolic acids and catechins. Free Radic Biol Med 2006;40:570-80.

25. Nirmala JG, Celsia E, Swaminathan A, Narendhirakannan RT, Chatterjee S. Cytotoxicity and apoptotic cell death induced by Vitis vinifera peel and seed extracts in A431 skin cancer cells. Cytotechnology 2018;70:537-554.

26. Akpolat M, Topcu-Tarladacalisir Y, Uz YH, Sapmaz-Metin M, Kizilay G. Kanser tedavisinde curcuminin yeri. Yeni Tip Dergisi 2010;27:142-47.
27. Sur S, Panda CK. Molecular aspects of cancer chemopreventive and therapeutic efficacies of tea and tea polyphenols. Nutrition 2017;4344:8-15.

28. Bahramsoltani R, Ebrahimi F, Farzai MH, Baratpourmoghaddam A, Borkani PA, Rostamiasrabadi P, et al. Dietary polyphenols for atherosclerosis: A comprehensive review and future perspectives. Crit Rev Food Sci Nutr 2019;59:114-32.

29. Castaner O, Covas MI, Khymenets O, Nyyssonen K, Konstantinidou V, Zunft HF, et al. Protection of LDL from oxidation by olive oil polyphenols is associated with a downregulation of CD40-ligand expression and its downstream products in vivo in humans. Am J Clin Nutr 2012;95:1238-44.

30. Wicinski M, Malinowski B, Weclewicz MM, Grezesk E, Grzesk G. Anti-atherogenic properties of resveratrol: 4-week resveratrol administration associated with serum concentrations of SIRT1, adiponectin, S100A8/A9 and VSMCs contractility in a rat model. Exp Ther Med 2017;13:2071-8.

31. Xu Y, Xu S, Koroleva M, Zhang S, Si S, Jin ZG. Tannic acid as a plant-derived polyphenol exerts vasoprotection via enhancing KLF2 expression in endothelial cells. Sci Rep 2017;7:6686.

32. International Diabetes Federation: The IDF consensus worldwide definitionof the metabolic syndrome 2005. http://www.idf.org/ webdata/docs/Metabolic_syndrome_definition.pdf.

33. Grosso G, Stepaniak U, Micek A, Stefler D, Bobak M, Pajak A. Dietary polyphenols are inversely associated with metabolic syndrome in Polish adults of the HAPIEE study. Eur J Nutr 2017;56:1409-20.

34. Basu A, Sanchez K, Leyva MJ, Wu M, Betts NM, Aston CE, et al. Green tea supplementation affects body weight,lipids, and lipid peroxidation in obese subjects with metabolic syndrome. J Am Coll Nutr 2010;29:31-40.

35. Selmanovic S, Beganlic A, Salihfendic N, Ljuca F, Softic A, Smajic E. Therapeutic effects of curcumin on ultrasonic morphological characteristics of liver in patients with metabolic syndrome. Acta Inform Med 2017;25:169-74.

36. Sung HY, Hong CG, Suh YS, Cho HC, Park JH, Bae JH, et al. Role of (-)-epigallocatechin-3-gallate in cell viability, lipogenesis, and retinol-binding protein 4 expression in adipocytes. Naunyn-Schmied Arch Pharmacol 2010;382:303-10.

37. Gregor MF, Hotamisligil GS. Inflammatory mechanisms in obesity. Annu Rev Immunol 2011;29:415-45.

38. Williamson G. The role of polyphenols in modern nutrition. Nutr Bull 2017;42:226-35.

39. Grassi D, Desideri G, Necozione S, Lippi C, Casale R, Properzi G et al. Blood pressure is reduced and insulin sensitivity increased in glucose-intolerant hypertensive subject safter 15 days of consuming high-polyphenol dark chocolate. J Nutr 2008;138:1671-6.

40. Serban MC, Sahebkar A, Zanchetti A, Mikhailidis DP, Howard G, Antal D, et al. Effects of quercetin on blood pressure: a systematic review and meta-analysis of randomized controlled trials. J Am Heart Assoc 2016;5. pii: e002713.

41. Daglia M. Polyphenols as antimicrobial agents. Curr Opin Biotechnol 2012;23:174-81.

42. Baharfar R, Azimi R, Mohseni M. Antioxidant and antibacterial activity of flavonoid-, polyphenol and anthocyanin-rich extracts from 
Thymuskotschyanusboiss\&hohen aerial parts. J Food Sci Technol 2015;52:6777-83.

43. Seo DJ, Choi C. Inhibitory mechanism of five natural flavonoids against murine norovirus. Phytomedicine 2017;30:59-66.

44. Karaosmanoglu H, Soyer F, Ozen B, Tokatlı F. Antimicrobial and antioxidant activities of Turkish extra virgin olive oils. J Agric Food Chem 2010;58:8238-45.

45. Avila JAD, Garcia JR, Aguilar GAG, de la Rosa LA. The antidiabetic mechanisms of polyphenols related to increased glucagon-like peptide-1 (GLP1) and insulin signaling. Molecules 2017;22.

46. Bozzetto L, Annuzzi G, Pacini G, Costabile G, Vetrani C, Vitale M, et al. Polyphenol-rich diets improve glucose metabolism in people at high cardiometabolic risk: a controlled randomised intervention trial. Diabetologia 2015;58:1551-60.
47. Tuzcu Z, Orhan C, Sahin N, Juturu V, Sahin K. Cinnamon polyphenol extract inhibits hyperlipidemia and inflammation by modulation of transcription factors in high-fat diet-fed rats. Oxid Med Cell Longev 2017;2017:1583098.

48. Vayalil PK. Date fruits (Phoenix dactylifera Linn): An emerging medicinal food.Crit Rev Food Sci Nutr 2012;52:249-71.

49. Ohkawara T, Takeda H, Nishihira J. Protective effect of chlorogenic acid on the inflammatory damage of pancreas and lung in mice with 1-arginine-induced pancreatitis. Life Sci 2017;190:91-6.

50. da Costa GF, Santos IB, de Bem GF, Cordeiro VSC, da Costa CA, de Carvalho LCRM, et al. The beneficial effect of anthocyanidinrich vitis vinifera L. grape skin extract on metabolic changes induced by high-fat diet in mice involves antiinflammatory and antioxidant actions. Phytother Res 2017;31:1621-32. 